\title{
New Design Method of UWB Microstrip Filters Using Adaptive Genetic Algorithms with Defected Ground Structures
}

\author{
Amir Reza Dastkhosh, ${ }^{1}$ Gholamreza Dadashzadeh, ${ }^{2}$ and Mohammad Hossein Sedaaghi ${ }^{1}$ \\ ${ }^{1}$ Faculty of Electrical Engineering, Sahand University of Technology, P.O. Box 51335-1996, Tabriz, Iran \\ ${ }^{2}$ Faculty of Electrical Engineering, Shahed University, Tehran 3319118651, Iran \\ Correspondence should be addressed to Amir Reza Dastkhosh, amir_reza_dastkhosh@yahoo.com
}

Received 9 August 2009; Revised 5 January 2010; Accepted 6 March 2010

Academic Editor: Safieddin Safavi-Naeini

Copyright (C) 2010 Amir Reza Dastkhosh et al. This is an open access article distributed under the Creative Commons Attribution License, which permits unrestricted use, distribution, and reproduction in any medium, provided the original work is properly cited.

\begin{abstract}
The effects of adaptive genetic algorithms (AGAs) and defected ground structures (DGSs) on performance optimization of tapered microstrip filter are investigated. The proposed structure achieves an ultra wide stopband with high attenuation within a small surface area, as well as $45 \%$ smaller size, in comparison with conventional filters. The parameters of the filter are optimized using in-home AGA code. In the proposed AGA algorithm, the crossover and mutation probabilities are adaptively changed according to the value of individual fitness. Then by utilizing the proposed DGS, a compact S-band lowpass filter with ultra-wide spurious free window is obtained. The proposed filter achieves an insertion loss of $0.8 \mathrm{~dB}$ from $\mathrm{DC}$ up to $4 \mathrm{GHz}$ and $21 \mathrm{~dB}$ rejection in the stopband from 4.3 up to $60 \mathrm{GHz}$. The fabricated and measured results exhibit good agreement with the simulated results. They demonstrate that combining AGA and DGS yields best possible response for this group of filters.
\end{abstract}

\section{Introduction}

In practice, high-performance microwave filters with minimized size and weight are playing an important role for design and fabrication of high-efficiency miniaturized microwave systems. Recently, due to high demand for broad band services, design of such miniaturized wide and ultrawide stopband filters for interference cancellation (by means of out of-band signal suppression) is gaining more and more attention [1-4]. Nonuniform transmission lines (NUTLs) play an important role in microwave circuits. Applications include impedance transformation and matching, filters, and directional couples. The nonuniform line is traditionally analyzed in the frequency domain [5-7].

Genetic algorithms are widely employed in various fields such as optimal engineering designs. They have been successfully applied to finding the global optimum in a variety of unimodal domains. Parameter control methods are classified as deterministic and adaptive. Deterministic systems employ fixed, predefined parameters for GA. On the other hand, adaptive control uses feedback from the search process to find out how the parameter values change $[8,9]$.
Many researchers have proposed and demonstrated electromagnetic bandgap (EBG) microstrip structures to achieve compact and wide frequency stopband [10-12]. Also recently, DGSs have become one of the most interesting areas of research in modern communication systems [13-16]. The DGS was first proposed by Kim et al. [17]. The microstrip line with DGS patterns in the ground plane has the stopband characteristics due to the equivalent effective inductance of DGS. It modifies the characteristics of the transmission line such as line capacitance and inductance.

However none of these papers presents a combined rigorous method to fully design and optimize new filters. We emphasize the new idea of optimization, analysis, and synthesis on nonuniform transmission lines using adaptive genetic algorithms and DGSs design methods. In sum, by using adaptive genetic algorithms and DGSs, characteristics and size of a tapered microstrip filter can be optimized. The new method yields a microstrip filter with high rejection in the stopband and sharp transition with reduced insertion loss in passband. These features can be used in filter applications to eliminate unwanted frequencies and to reduce the physical size of a microstrip circuit. Conventional filtering 


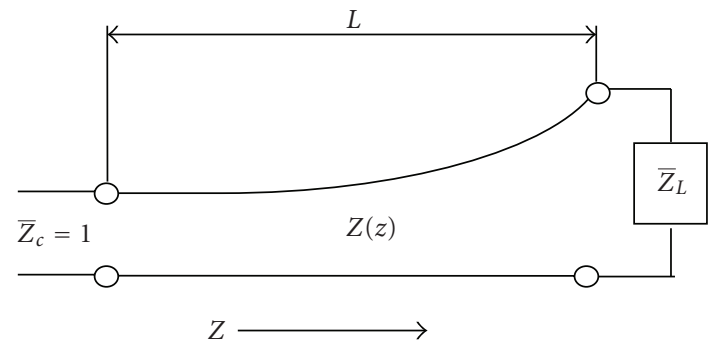

(a)

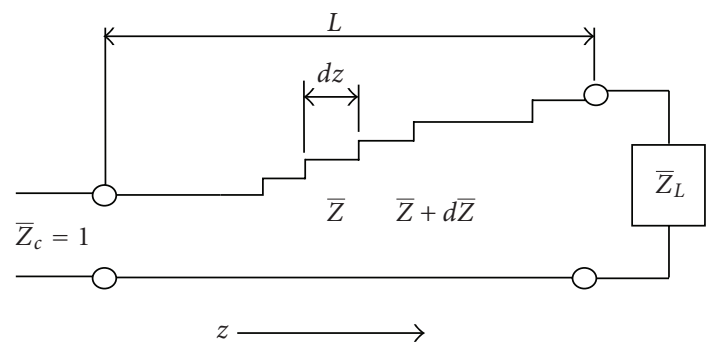

(b)

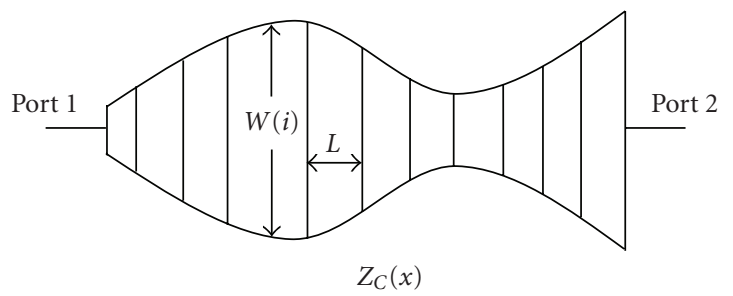

(c)

Figure 1: (a) A portion of nonuniform transmission line. (b) Approximation of part (a). (c) Its tapered structure.

methods in microstrip designs with shunt stubs (or steppedimpedance lines) present spurious harmonic frequencies in stopband and occupy valuable circuit layout area. An Sband lowpass filter is designed and measured as an example of the superiority of the proposed method. Firstly, AGA is applied to find the optimum parameters according to the desired attenuation. Then DGSs are employed to improve the results. Also we have proposed a high-performance and compact lowpass filter structure with ultra-wide stopband using adaptive genetic algorithms and new DGS configurations.

\section{Design of Tapered Microstrip Filter}

The continuously tapered line can be built using incremental sections of length $d z$ with an impedance change $\Delta Z(z)$ from one section to next one. Figure 1(a) illustrates a nonuniform transmission line terminating to a normalized load impedance $\overline{Z_{L}}$ and Figures 1(b) and 1(c) show the continuously tapered line.

An approximation theory, based on small reflections, is used to predict the reflection coefficient response as

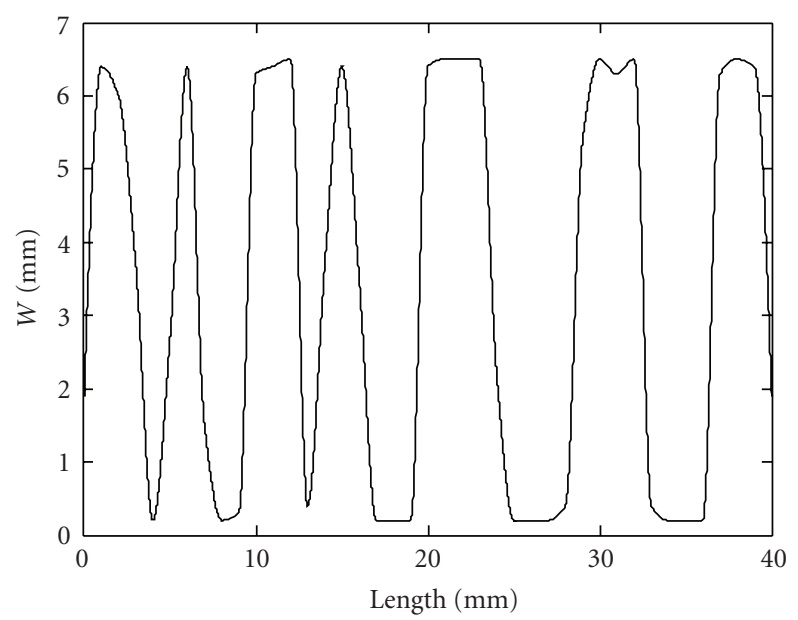

(a)

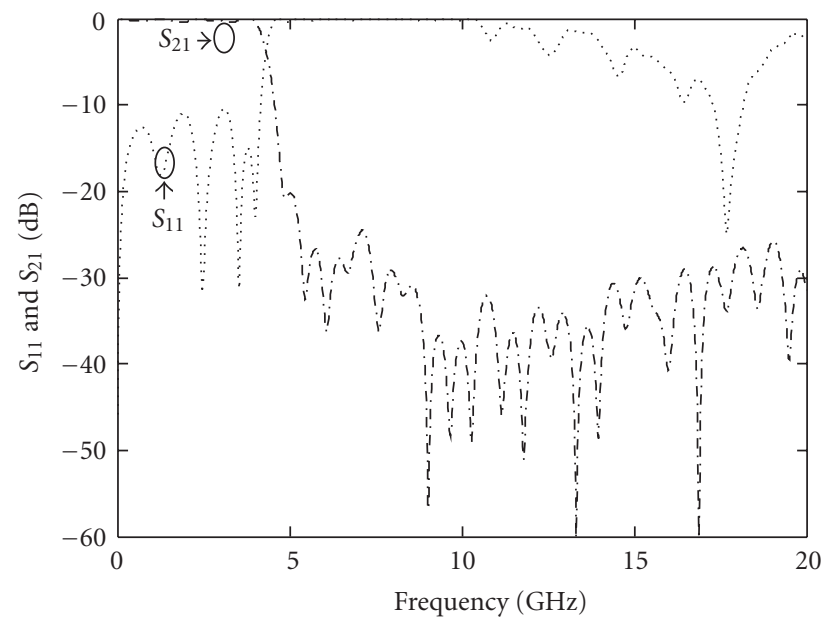

(b)

Figure 2: (a) Width $(W)$ of the optimized filter versus its length (L). (b) Simulated frequency response of the filter using CST 2008 (Optimization I).

a function of impedance taper $Z(z)$ as follows (see [18]):

$$
\begin{gathered}
\Gamma_{i}(2 \beta)=\frac{1}{2} \int_{0}^{L} e^{-j 2 \beta z} \frac{d(\ln \bar{Z})}{d z} d z, \\
\left|\Gamma_{i}\right|^{2}=1-|H(u)|^{2}, \quad u=\frac{\beta L}{\pi}=\frac{2 L}{\lambda}, H=\frac{V_{\text {out }}}{V_{\text {in }}},
\end{gathered}
$$

where $L$ is the length of the filter. Equation (1) is the Fourier transform of the impedance profile function $d(\ln \bar{Z}) / d z$. Consequently, the inverse transform of (1) is an expression which describes the spatial variation of the impedance profile $Z(x)$ as a function of the input reflection coefficient $\Gamma_{i}(2 \beta)$ as explained below:

$$
\frac{1}{2} \frac{d \ln (\bar{Z})}{d z}=\frac{1}{2 \pi} \int_{-\infty}^{+\infty} e^{j 2 \beta z} \Gamma_{i}(2 \beta) 2 d \beta .
$$

Equation (2) is the synthesis one. An approximate answer is achieved using numerical integration. Then the characteristic impedance function $\bar{Z}$ can be obtained with a tapered 


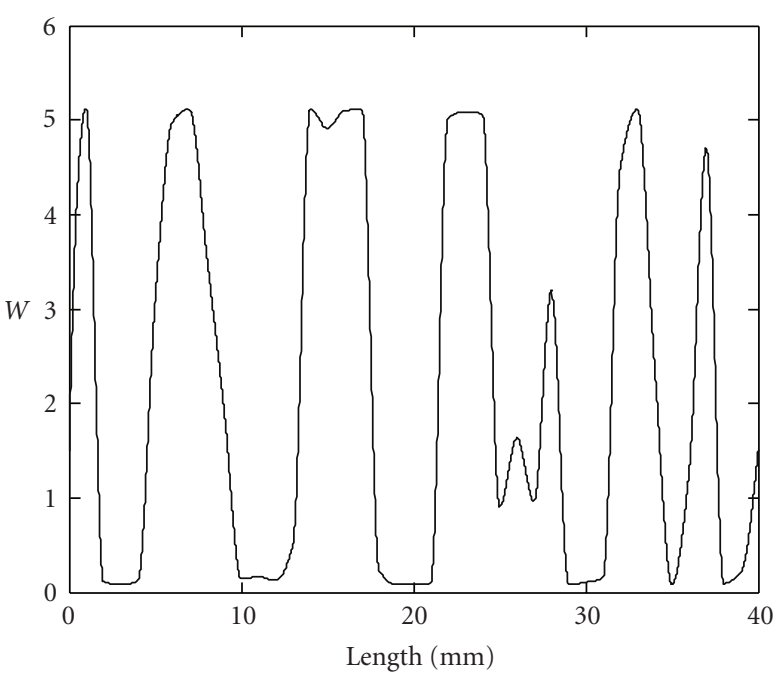

(a)

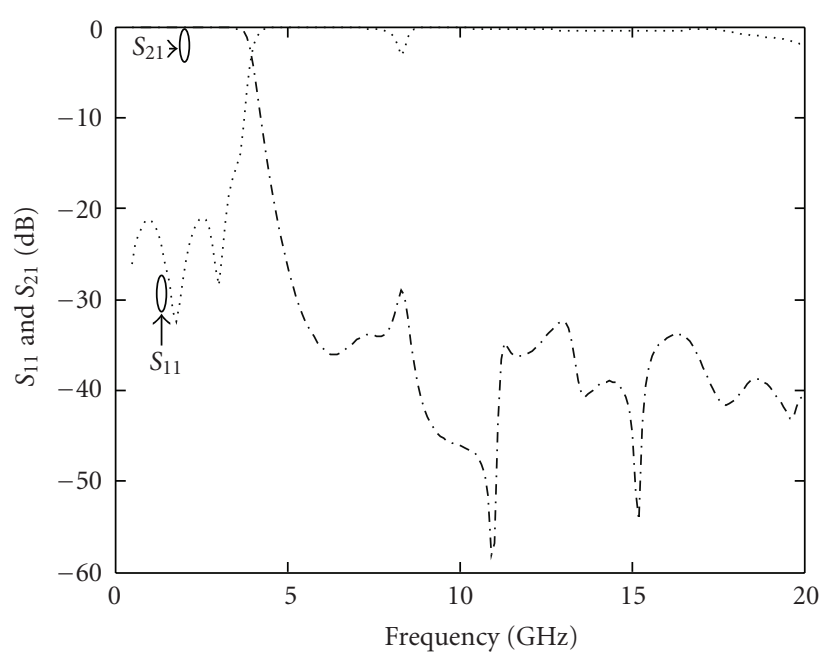

(b)

FIGURE 3: (a) Width $(W)$ of the optimized filter versus its length $(L)$. (b) Simulated frequency response of the filter using Ansoft Designer 4 (Optimization II).

microstrip filter as a primary structure. A lossless nonuniform transmission line may be considered as a limiting case where the number of nonuniform transmission lines $(N)$ in stepped impedance transformers becomes infinitely large and, at the same time, the maximum length of each nonuniform transmission line-section approaches to zero, provided that the total length $L$ of the stepped impedance transformer is equal to nonuniform transmission line. To analyze the filter, its structure is divided into a number of short segments based on the length of the filter as illustrated in Figure 1. The structure can be simulated more precisely using tapered sections. Therefore, the tapered sections are used for filter modeling $[19,20]$.

\section{Adaptive Genetic Algorithms}

In this section the optimization of the filter specifications using adaptive genetic algorithms [21-23] is introduced. The behavior of genetic algorithms is strongly influenced by the balance between exploration and exploitation. The GA control parameter settings, such as mutation and crossover probabilities (denoted by $P_{m}$ and $P_{c}$, resp.) and the population size, are key factors in the determination of the exploitation versus exploration tradeoff. If poor settings are used, the exploration/exploitation balance may not be reached in a profitable way; the GA performance is severely affected due to the possibility of premature convergence. Finding robust control parameters is not a trivial task since their interaction with GA performance is a complex relationship and the optimal ones are problem dependent. Therefore, adaptive GAs are considered where they dynamically adjust the selected control parameters or genetic operators during the course of evolving a problem solution. The significance of $P_{c}$ and $P_{m}$ in controlling GA performance has been acknowledged in GA research [24-26].
$P_{c}$ controls the rate at which a solution is subjected to crossover. The higher the value of $P_{c}$, the quicker the new solutions are introduced into the population. However, as $P_{c}$ increases, solutions can be disrupted faster than the selection can exploit them. Typical values of $P_{c}$ are in the range 0.51.0. Mutation is another operator to restore genetic material. Larger values of $P_{m}$ transform GA into a purely random search algorithm while a little mutation is only required to prevent the premature convergence of the GA into a suboptimal solution. A good selection of $P_{c}$ and $P_{m}$ can lead to an easier search for good solution. $P_{m}$ and $P_{c}$ depend on the fitness of the solutions and are zero for the solution with the maximum fitness. Therefore, they are adapted as follows:

$$
\begin{aligned}
& P_{c}=k_{1} \frac{f_{\mathrm{Max}}-f^{\prime}}{f_{\mathrm{Max}}-\bar{f}} \\
& \left(\text { if } f^{\prime} \leq \bar{f} \Longrightarrow P_{c}=k_{3}, k_{1}=k_{3}=1\right), \\
& P_{m}=k_{2} \frac{f_{\mathrm{Max}}-f}{f_{\mathrm{Max}}-\bar{f}} \\
& \left(\text { if } f \leq \bar{f} \Longrightarrow P_{m}=k_{4}, k_{2}=k_{4}=0.5\right) .
\end{aligned}
$$

$\bar{f}, f_{\text {Max }}, f$, and $f^{\prime}$ are the average fitness value of the population, the maximum of the fitness, the fitness of each member of the population, and the larger fitness of the solutions to be crossed, respectively. Also the normalized fitness function is selected as

$$
F=100 \times\left(\frac{2}{3}\left|S_{11}\right|+\frac{1}{3}\left|S_{21}\right|\right),
$$

where $S_{11}$ and $S_{21}$ are the worst points in the stopband and passband regions, respectively.

As the signal's frequency applied to millimeter-wave integrated circuit is steadily increased, some characteristic 


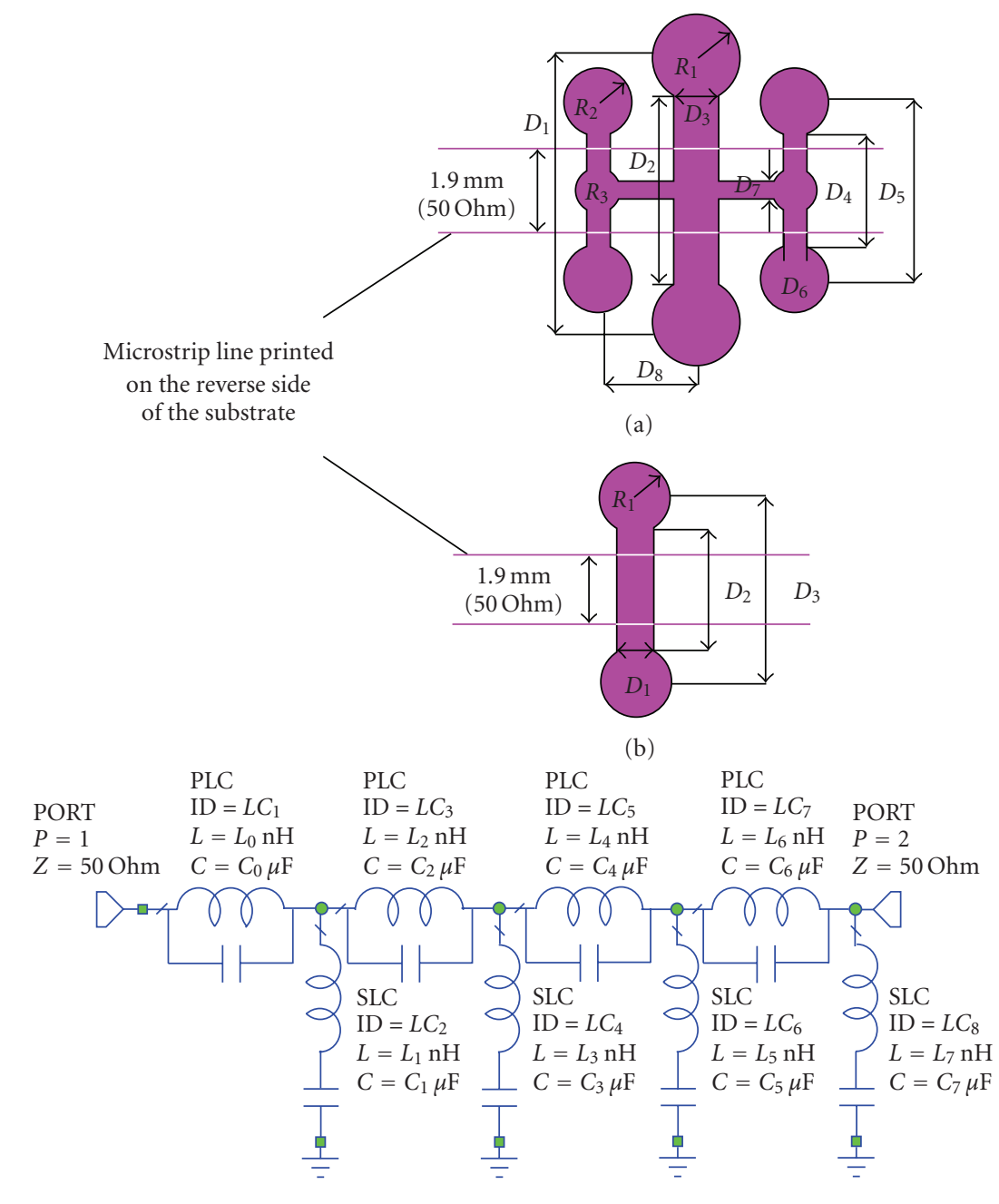

(c)

FIGURE 4: Required DGS structures. (a) and (b) DGS (1) \& DGS (2). (c) Resulting circuit model. Solid lines indicate that the slots are etched in the ground plane of the microstrip.

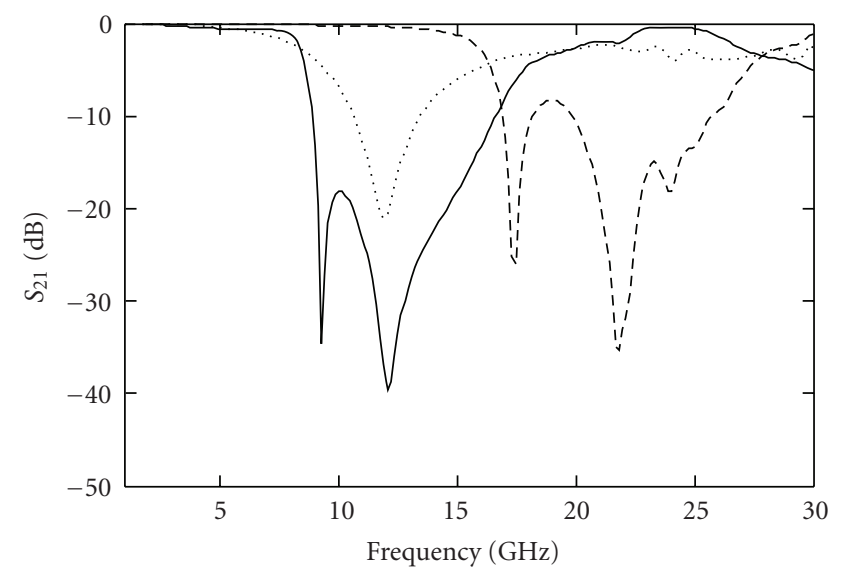

$\begin{array}{ll}\text { …. } & \text { DGS2 (Case A) } \\ \text { - } & \text { DGS1 (Case A) } \\ \text { - - } & \text { DGS1 (Case B) }\end{array}$

FIGURE 5: Frequency responses of DGS structures. frequency may be reached at which undesirable effects occur. The same is true for monolithic integrated circuits. For a sufficiently wide microstrip line, a transverse-resonant mode exists which can also be coupled strongly to the quasiTEM microstrip mode. Therefore, because of fabrication technology and in order to avoid the excitation of higherorder modes in a microstrip, the maximum and minimum values of the microstrip widths are limited to $0.1-6.5 \mathrm{~mm}$ [27]. By this condition, a wider stopband filter can be obtained. The width of the microstrip feed line is fixed at $W_{1}=1.9 \mathrm{~mm}$ to achieve $50 \Omega$ impedance. The total length of the filter is assumed to be a wavelength. The number of NUTL-sections is selected to be 40 .

The optimization algorithm is employed to obtain the lowpass filter with the determined specifications. The optimization variables for the algorithm are widths of nonuniform microstrip transmission line. Distinctive optimized structures are investigated using two different optimization goals and denoted Optimization I \& II, respectively. The values for width and the simulated frequency responses are 


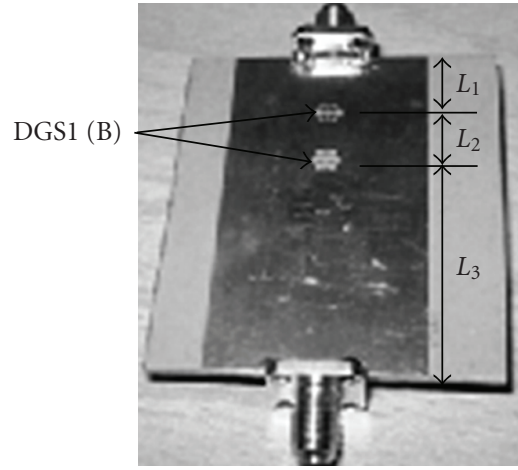

(a)

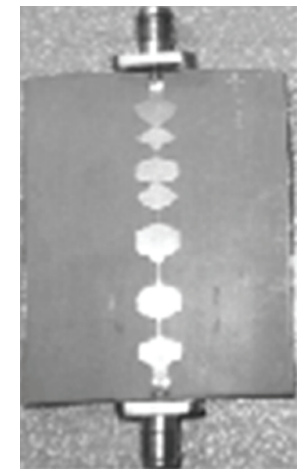

(b)

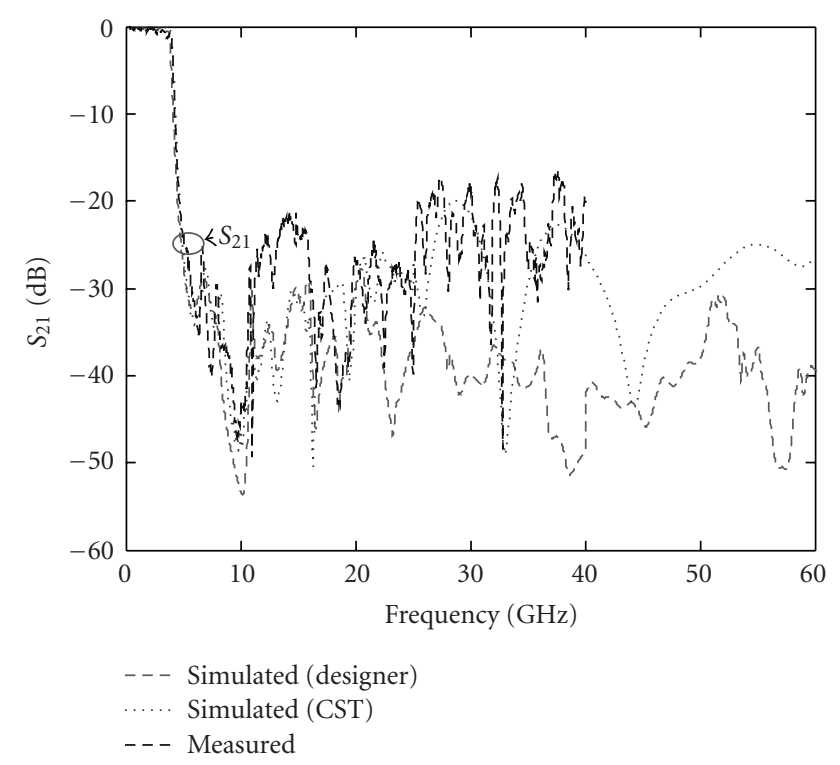

(c)

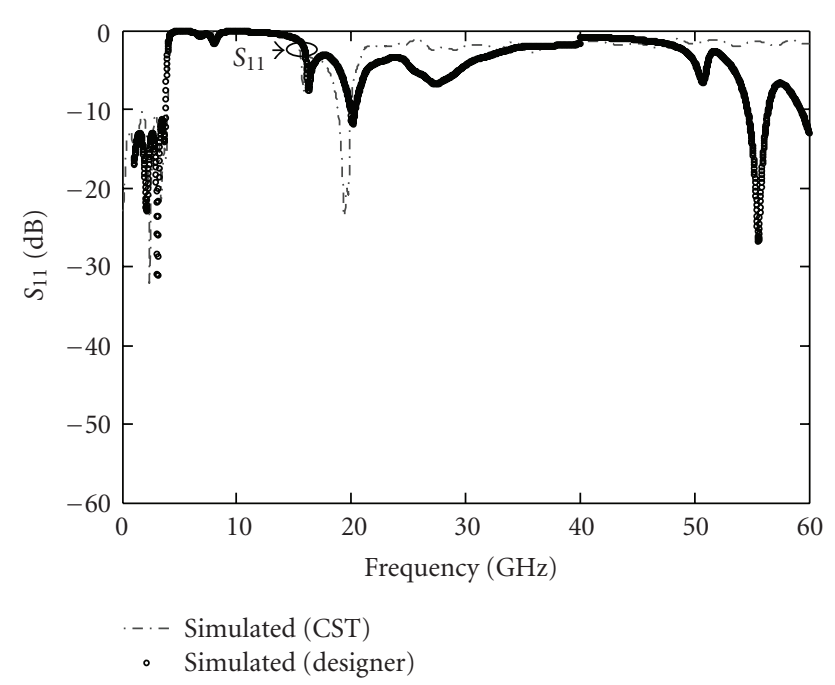

(d)

FIGURE 6: Fabricated tapered microstrip filter optimized by DGSs (Optimization I): (a) Top, (b) Bottom, (c), (d): Frequency responses (Simulated and Measured) $L_{1}=10.5, L_{2}=18.5$, and $L_{3}=21$.

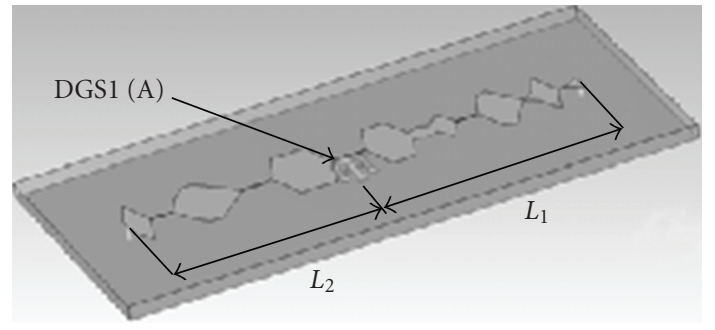

(a)

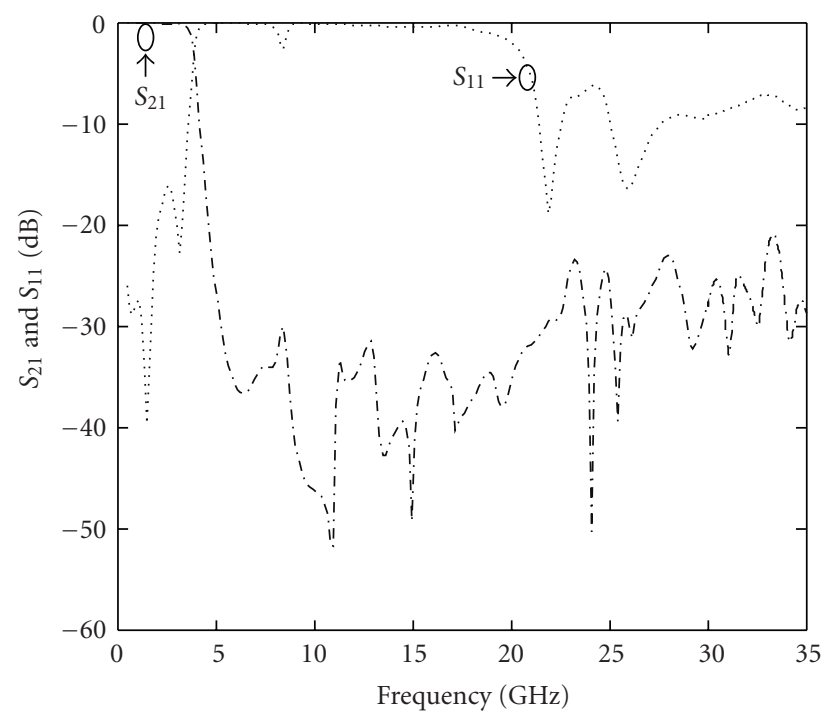

(b)

FIGURE 7: Simulated tapered microstrip filter optimized by DGSs (Optimization II): (a) Structure and (b) frequency responses $\left(L_{1}=\right.$ $\left.L_{2}=20\right)$.

illustrated in Figures 2 and 3, respectively, and are simulated on a $1.27 \mathrm{~mm}$ Rogers TMM substrate with a dielectric constant of 6 . The structures are simulated with the commercial softwares, Ansoft Designer 4 and CST Microwave Studio 2008. It is seen that the optimized insertion loss for a lowpass filter is more than $-0.3 \mathrm{~dB}$ from $\mathrm{DC}$ up to $4 \mathrm{GHz}$ and the rejection band is better than $20 \mathrm{~dB}$ in $4.7-20 \mathrm{GHz}$ range.

\section{Defected Ground Structures}

The defected ground structure is an etched defect in the ground plane of a microstrip circuit. This disturbance changes the characteristics of a transmission line. It disturbs the current distribution in the ground plane. By changing the geometric dimensions, shapes or places of DGSs, it is possible to modify the effective capacitance and inductance of the transmission line. The DGSs investigated in this work and their equivalent circuits are demonstrated in Figure 4. The proposed structure in this paper is based on a combination of tapered etched holes and periodic DGSs.

As it is understood from the frequency responses, DSGs need to be designed as a band-reject filter to achieve a reasonable functionality. The best values for dimensions are found using Ansoft Designer 4. The circuit parameters are 


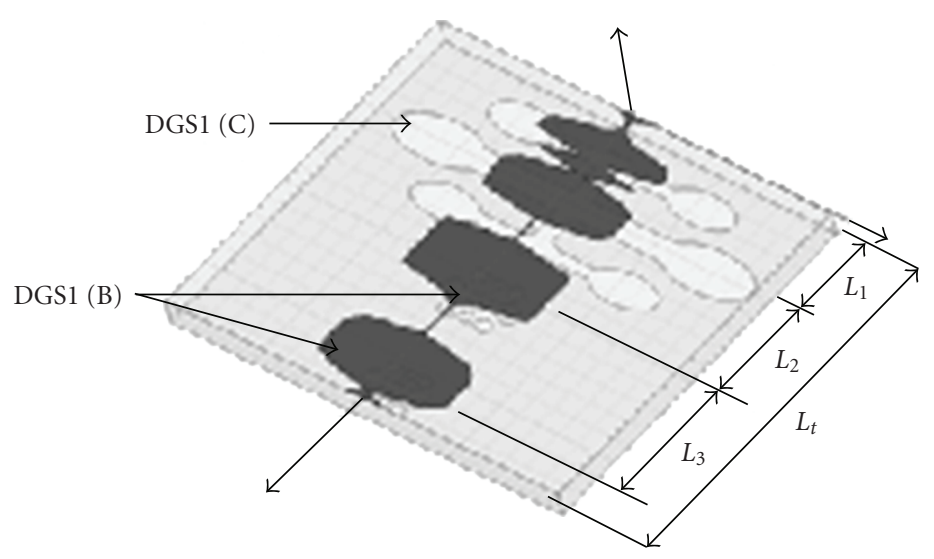

(a)
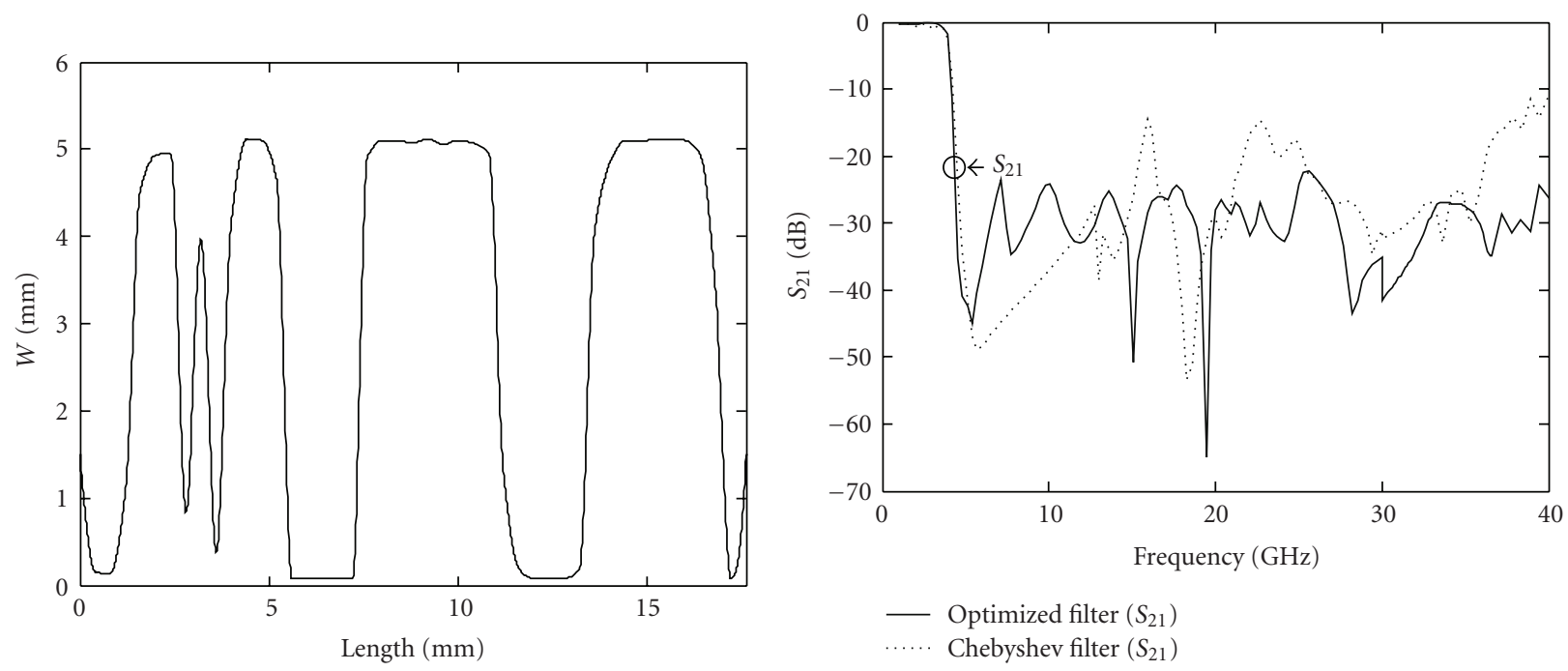

(b)

(c)

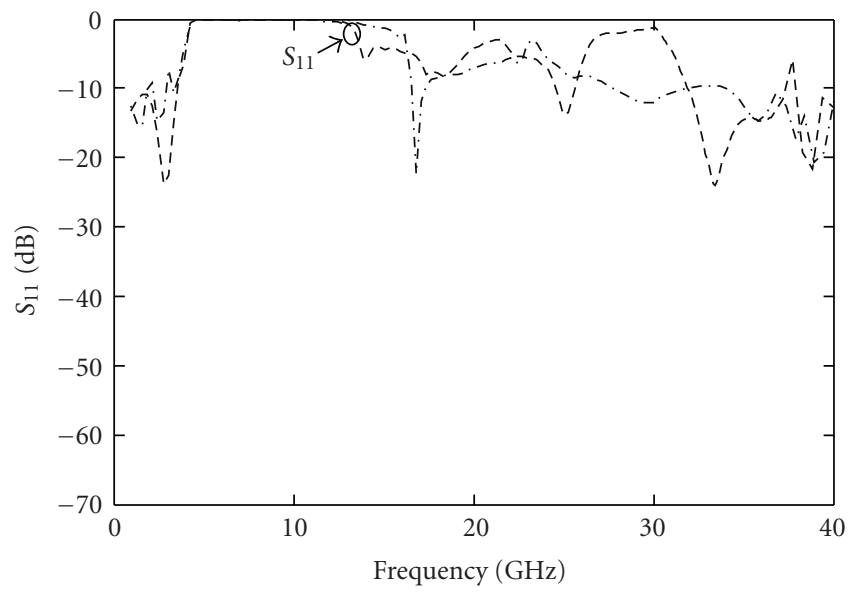

- - Optimized filter $\left(S_{11}\right)$

-..- Chebyshev filter $\left(S_{11}\right)$

(d)

FIGURE 8: Simulated optimized compact filter: (a) structure (b) width values of the optimized filter (c), (d) frequency response $\left(L_{t}=17, L_{1}=\right.$ $\left.4, L_{2}=6.2, L_{3}=5.5\right)$. 


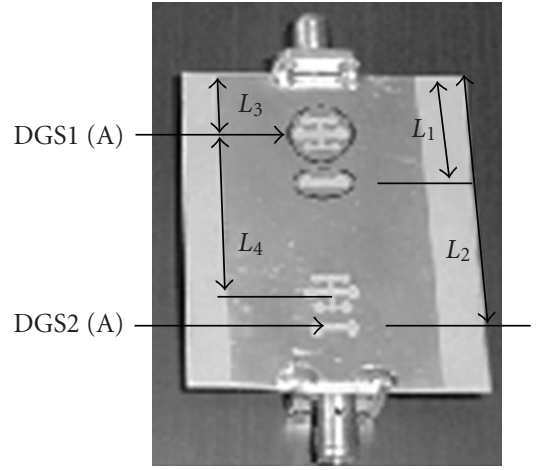

(a)

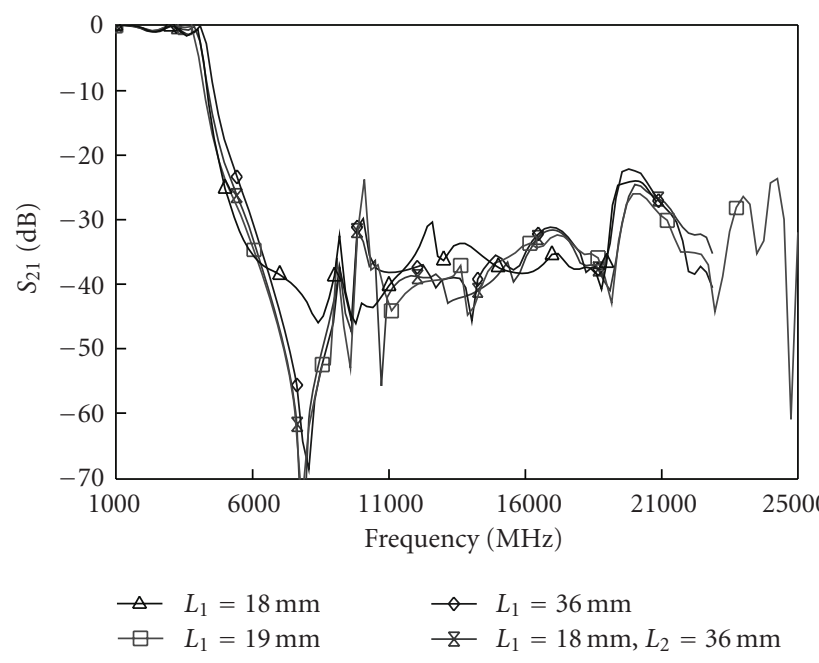

(b)

FIGURE 9: Setting up the cutoff frequency of the optimized filter: (a) the structure and (b) the frequency responses when $\left(L_{3}=5, L_{4}=\right.$ 25).

extracted from an electromagnetic simulation employing a Butterworth bandstop filter response. Figure 5 depicts the frequency responses of the DGSs. Various dimensions of the required DGSs have been listed in Table 1.

\section{Experimental Results}

In order to show the effectiveness of the proposed method and the accuracy of the model, we present experimental results. Figures 6 and 7 illustrate photos of the fabricated filter, the dimensions of structures, and their frequency responses. The DGS elements are cascaded in order to realize wider stopband with very sharp edge filter. The measurements are performed with an Agilent-8722ES network analyzer. CST 2008 and Ansoft Designer 4 use different numerical techniques to simulate the structures. Variations in the measured performance are mainly due to imprecise fabrication by a milling machine, the effect of SMA connectors, and the nonideal soldering. Also, because of the limitations in practical implementation, we were forced to use Rogers RT/duroid 6006 (TM) with a dielectric constant
TABle 1: Dimensions (in $\mathrm{mm}$ ) of DGSs with different resonant frequencies.

\begin{tabular}{lcc}
\hline DGS structures & DGS $(1)$ & DGS $(2)$ \\
\hline Case A & $R_{1}=1, R_{2}=0.75, R_{3}=0.5$ & $R_{1}=1$ \\
& $D_{1}=6, D_{2}=4.3, D_{3}=1$, & $D_{1}=1$ \\
& $D_{4}=2.6, D_{5}=4, D_{6}=0.5$, & $D_{2}=3.3$ \\
& $D_{7}=0.4, D_{8}=2.2$ & $D_{3}=5$ \\
\hline & $R_{1}=0.5, R_{2}=0.4$, & \\
& $R_{3}=0.25, D_{1}=3.2$, & \\
& $D_{2}=2.2, D_{3}=0.5$, \\
& $D_{4}=1.3, D_{5}=2.2$, \\
& $D_{6}=0.25, D_{7}=0.2$, & \\
& $D_{8}=1.1$ & \\
\hline & $R_{1}=2.25, R_{2}=1.8$, & \\
Case B C & $R_{3}=1.2, D_{1}=14, D_{2}=9.6$, & \\
& $D_{3}=1.1, D_{4}=5.8, D_{5}=9.4$, \\
& $D_{6}=0.55, D_{7}=0.9, D_{8}=.5$ &
\end{tabular}

of 6.15. The simulation results show that the proposed structure has an ultra-wide stopband and its peak remains below the acceptable $-22 \mathrm{~dB}$ level from 4.5 up to $60 \mathrm{GHz}$.

The operational frequency range of the Agilent-8722ES vector network analyzer was $50 \mathrm{MHz}$ up to $40 \mathrm{GHz}$. Therefore, we were not able to measure the characteristics of the designed filter beyond $40 \mathrm{GHz}$. Also, only the $S_{21}$ measurements were accessible. However, as it can be seen from Figures 6 and 7, the simulation of the filter was performed up to $60 \mathrm{GHz}$ using CST 2008 and Ansoft Designer 4 softwares. The structures were simulated with loss-free substrates and the same results were obtained.

In another optimized structure, we present a more compact design of the ultra-wide stopband lowpass filter. Figure 8 illustrates the dimensions and frequency response of the optimized compact filter. According to its specifications, a conventional 10th-order Chebyshev stepped-impedance lowpass microstrip filter is designed and optimized by Agilent ADS 2008 to highlight the performance of the optimized filter. The conventional Chebyshev filter has $32 \mathrm{~mm}$ length while the optimized filter's length is less than a half-wavelength (i.e., $17 \mathrm{~mm}$ ). The optimized compact filter has sharper band edge and deeper and wider stopband.

In this case, the stopband peak is less than $-21 \mathrm{~dB}$ from 4.3 up to $60 \mathrm{GHz}$. As mentioned earlier, a compact deep attenuation and spurious-free filter is achieved by this method. The dimensions of the new DGS configuration are demonstrated in Figure 8(a) and Table 1.

One of the interesting results of using such structures is the ability to set up the cutoff frequency of these filters up to hundreds of $\mathrm{MHz}$ only by displacing a few millimeters of the DGSs with respect to their current positions as shown in Figure 9. The changes in characteristic impedance will shift the cutoff frequency. It is proportional to geometry, size, the amount, and displacement of DGSs. A varactor-loaded resonator can be implemented to design a tunable and highselective lowpass filter. 


\section{Conclusion}

An adaptive genetic algorithm is combined with nonuniform transmission line to optimize a tapered microstrip lowpass filter. With the DGS configurations, the proposed structure displays an ultra-wide stopband with high attenuation within a small circuit area. By applying adaptive genetic algorithms and DGSs, the performance of a tapered microstrip filter is significantly improved. Also, the proposed lowpass filters have wider and deeper stopband characteristics compared to those of conventional lowpass ones. The equivalent circuits and the related design procedures have been discussed in details. Numerical simulations using CST Microwave Studio 2008 and Ansoft Designer 4 show promising agreement with experiments. The measured data for fabricated DGS-based lowpass filters have fairly good insertion-loss characteristics. The designed filters have a $4 \mathrm{GHz}$ cutoff frequency and also a stopband attenuation less than $-21 \mathrm{~dB}$ from $4.3 \mathrm{GHz}$ up to $60 \mathrm{GHz}$. As a result now, one can design a desired compact filter, starting from a predefined structure.

\section{References}

[1] J. S. Hong and M. J. Lancaster, Microstrip Filters for RF/Microwave Applications, John Wiley \& Sons, New York, NY, USA, 2001.

[2] J.-Z. Gu, W.-Y. Yin, R. Qian, C. Wang, and X.-W. Sun, "A wideband EBG structure with 1D compact microstrip resonant cell," Microwave and Optical Technology Letters, vol. 45, no. 5, pp. 386-387, 2005.

[3] M. K. Mandal and S. Sanyal, "Compact wideband bandpass filter," IEEE Microwave and Wireless Components Letters, vol. 16, no. 1, pp. 46-48, 2006.

[4] S. Sun and L. Zhu, "Capacitive-ended interdigital coupled lines for UWB bandpass filters with improved out-of-band performances," IEEE Microwave and Wireless Components Letters, vol. 16, no. 8, pp. 440-442, 2006.

[5] N. H. Younan, B. L. Cox, C. D. Taylor, and W. D. Prather, "Exponentially tapered transmission line antenna," IEEE Transactions on Electromagnetic Compatibility, vol. 36, no. 2, pp. 141-144, 1994.

[6] C. E. Baum and J. M. Lehr, "Tapered transmission-line transformers for fast high-voltage transients," IEEE Transactions on Plasma Science, vol. 30, no. 5, pp. 1712-1721, 2002.

[7] L. A. Hayden and V. K. Tripathi, "Nonuniformly coupled microstrip transversal filters for analog signal processing," IEEE Transactions on Microwave Theory and Techniques, vol. 39, no. 1, pp. 47-53, 1991.

[8] A. E. Eiben, R. Hinterding, and Z. Michalewicz, "Parameter control in evolutionary algorithms," IEEE Transactions on Evolutionary Computation, vol. 3, no. 2, pp. 124-141, 1999.

[9] D. Thierens, "An adaptive pursuit strategy for allocating operator probabilities," in Proceedings of the ACM Genetic and Evolutionary Computation Conference (GECCO '05), pp. 15391546, 2005.

[10] M. A. G. Laso, T. Lopetegi, M. J. Erro, D. Benito, M. J. Garde, and M. Sorolla, "Multiple-frequency-tuned photonic bandgap microstrip structures," IEEE Microwave and Wireless Components Letters, vol. 10, no. 6, pp. 220-222, 2000.
[11] C. C. Chiau, X. Chen, and C. Parini, "Multiperiod EBG structure for wide stopband circuits," IEE Proceedings, vol. 150, no. 6, pp. 489-492, 2003.

[12] Y.-C. Chen, A.-S. Liu, and R.-B. Wu, "A wide-stopband low-pass filter design based on multi-period taper-etched EBG structure," in Proceedings of the Asia-Pacific Microwave Conference Proceedings (APMC '05), vol. 3, pp. 2125-2127, Suzhou, China, December 2005.

[13] M. K. Mandal and S. Sanyal, "A novel defected ground structure for planar circuits," IEEE Microwave and Wireless Components Letters, vol. 16, no. 2, pp. 93-95, 2006.

[14] Y. Chung, S.-S. Jeon, D. Ahn, J.-I. Choi, and T. Itoh, "High isolation dual-polarized patch antenna using integrated defected ground structure," IEEE Microwave and Wireless Components Letters, vol. 14, no. 1, pp. 4-6, 2004.

[15] Z. Pan and J. Wang, "Design of the UWB bandpass filter by coupled microstrip lines with U-shaped defected ground structure," in Proceedings of the International Conference on Microwave and Millimeter Wave Technology (ICMMT'08), vol. 1, pp. 329-332, April 2008.

[16] T. Kido, H. Deguchi, M. Tsuji, and M. Ohira, "Compact high-performance planar bandpass filters with arbitrarilyshaped conductor patches and slots," in Proceedings of the 38th European Microwave Conference (EuMC '08), pp. 1165-1168, October 2008.

[17] C.-S. Kim, J.-S. Park, D. Ahn, and J.-B. Lim, "A novel 1-D periodic defected ground structure for planar circuits," IEEE Microwave and Wireless Components Letters, vol. 10, no. 4, pp. 131-133, 2000.

[18] R. E. Collin, Foundations for Microwave Engineering, McGrawHill, New York, NY, USA, 2nd edition, 2000.

[19] K. Lu, "An efficient method for analysis of arbitrary nonuniform transmission lines," IEEE Transactions on Microwave Theory and Techniques, vol. 45, no. 1, pp. 9-14, 1997.

[20] C. L. Edwards, M. L. Edwards, S. Cheng, R. K. Stilwell, and C. C. Davis, "A simplified analytic CAD model for linearly tapered microstrip lines including losses," IEEE Transactions on Microwave Theory and Techniques, vol. 52, no. 3, pp. 823830, 2004.

[21] M.-I. Lai and S.-K. Jeng, "Compact microstrip dual-band bandpass filters design using genetic-algorithm techniques," IEEE Transactions on Microwave Theory and Techniques, vol. 54, no. 1, pp. 160-168, 2006.

[22] E. Michielssen, J.-M. Sajer, S. Ranjithant, and R. Mittra, "Design of lightweight, broad-band microwave absorbers using genetic algorithms," IEEE Transactions on Microwave Theory and Techniques, vol. 41, no. 6-7, pp. 1024-1030, 1993.

[23] T. Nishino and T. Itoh, "Evolutionary generation of microwave line-segment circuits by genetic algorithms," IEEE Transactions on Microwave Theory and Techniques, vol. 50, no. 9, pp. 2048-2055, 2002.

[24] M. Srinivas and L. M. Patnaik, "Adaptive probabilities of crossover and mutation in genetic algorithms," IEEE Transactions on Systems, Man and Cybernetics, vol. 24, no. 4, pp. 656667, 1994.

[25] C.-L. Liu, Z.-Y. Wang, and Z. Bao, "A kind of adaptive genetic algorithm and it's application in model identification," in Proceedings of the 4th International Conference on Machine Learning and Cybernetics (ICMLC '05), vol. 5, pp. 2865-2869, August 2005.

[26] B. T. Skinner, H. T. Nguyen, and D. K. Liu, "Performance study of a multi-deme parallel genetic algorithm with adaptive 
mutation," in Proceedings of the 2nd International Conference on Autonomous Robots and Agents, Palmerston North, New Zealand, December 2004.

[27] T. C. Edwards and M. B. Steer, Foundations of Interconnect and Microstrip Design, John Wiley \& Sons, New York, NY, USA, 3rd edition, 2000. 

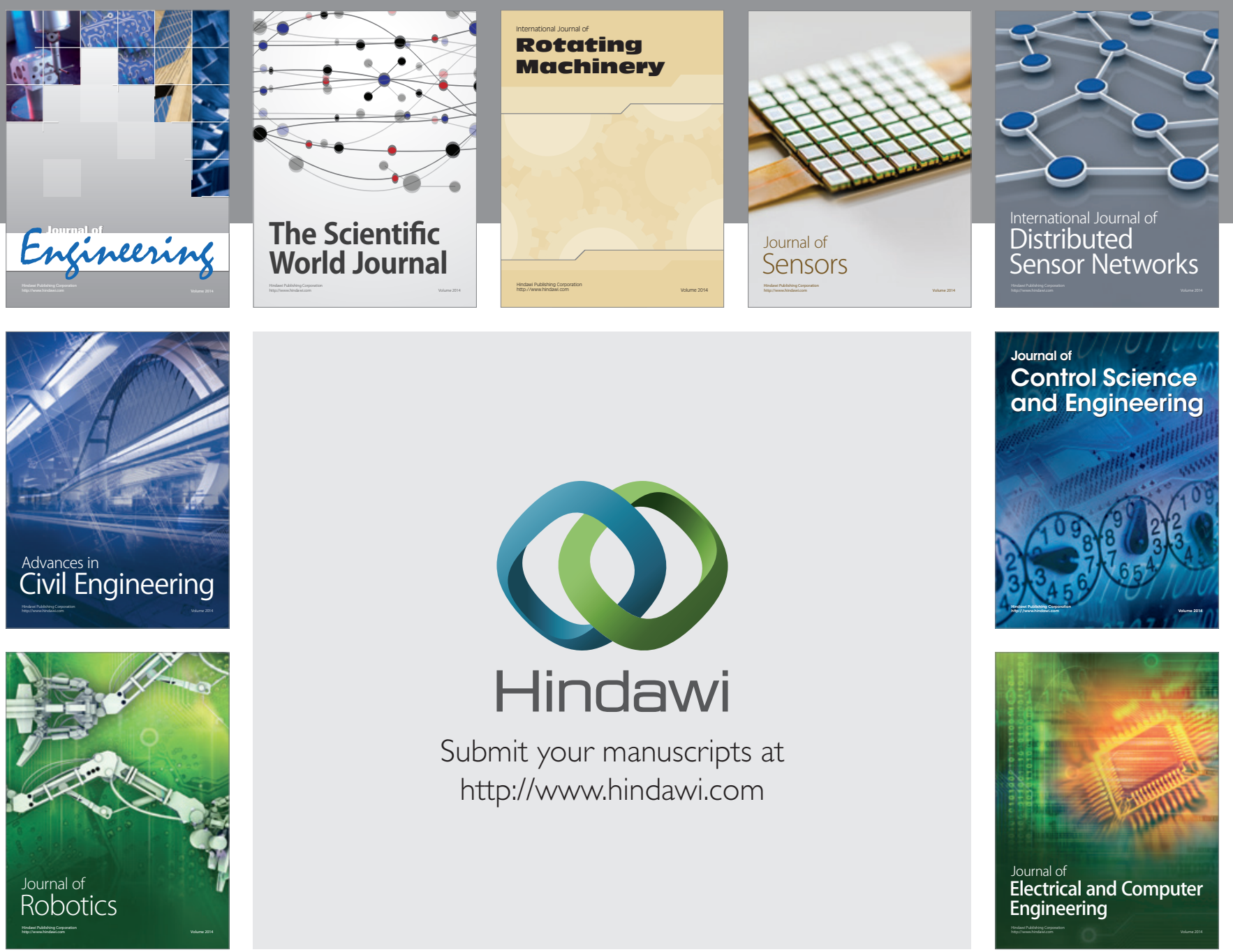

Submit your manuscripts at

http://www.hindawi.com
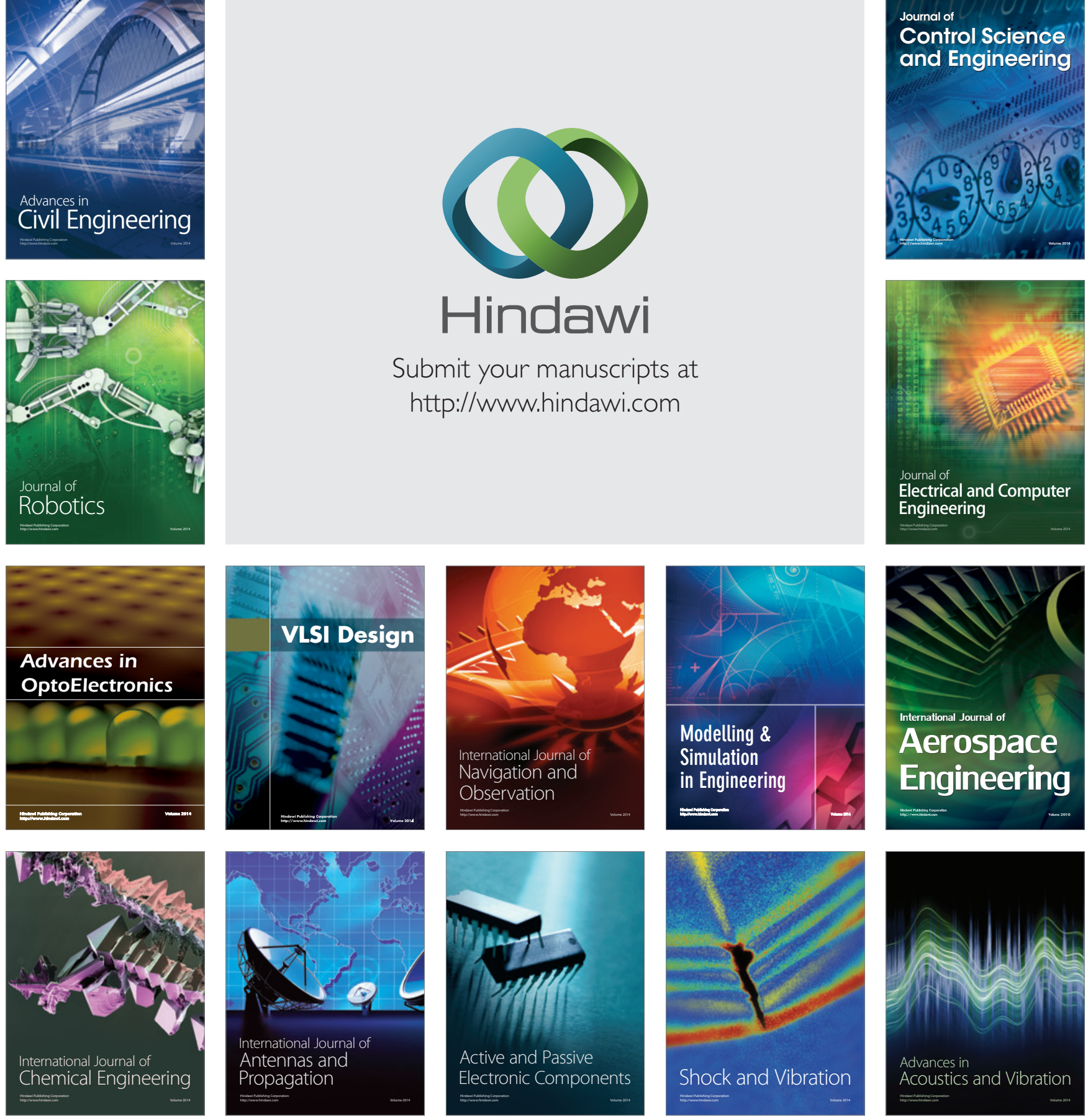